\title{
34
}

\section{A classification of health information systems security flaws}

\author{
D. Gritzalis a , I. Kantzavelou b, S. Katsikas a and A. Patel b \\ a Dept. of Mathematics, University of the Aegean, Samos GR-83200, Greece \\ b Dept. of Computer Science, University College Dublin, Belfield, Dublin 4, Ireland
}

The increasing interest of both business and government sectors in information security issues has led up to the collection of several security incidents in inventories. Most of these inventories pertain to specific environments; among them there exist inventories pertaining to health information systems. Information included in these inventories could be exploited by information systems developers, if a proper means for their effective processing is provided. Such a means is a recently proposed taxonomy scheme which classifies information system security flaws according to how, where and when they been introduced. In this paper, this taxonomy scheme has been used to classify health information system security flaws. Conclusions based on the results of this classification are drawn.

\section{SECURITY FLAWS AND RISK ANALYSIS}

Risk analysis is a fundamental part of the experience of all managers responding to a wide variety of situations. One of these situations deals with the countermeasures that should be taken, when a security flaw is identified in an automated information system, or in order that this flaw - or any foreseeable other - does not occur.

Security flaws, for the purposes of this paper, are defined as any conditions or circumstances that can result in denial of service, unauthorised disclosure, unauthorised destruction or unauthorised modification of data [Lan-81].

Given a relevant framework including the definition of information security, the role of information security risk analysis has been summarised as follows [Bad-89, Bad-90, Elo-93]:

- Risk analysis is essential for both the development of a security policy and for the refinement of such a policy, in order to define a protection plan for implementation.

- Risk analysis can be used as an effective means for getting senior management involved in information risk management, since the management of business risk is considered by management as a success factor.

- The cornerstone output of a risk analysis review is the identification of appropriate countermeasures for the identified risks.

However, most risk analysis methods are not of a formal nature. They are based on the test of hypotheses, on selected and - potentially - reliable heuristics, on the wide experience of 
several experts in a field, and on the knowledge of the experts in the information systems they review. In the best case, risk analysis reviews provide useful results; however, they cannot provide any formal guarantee, based on a objective cost-benefit scenario, that the proposed countermeasures are the best ones. Furthermore, the selection of the proposed countermeasures is a procedure which requires much more than processing simple what-if scenarios or even the use of rule-based expert systems. Finally, some countermeasures may not be included in the countermeasure data base, because of the rapid technological evolution.

In any case, risk analysis is an effective methodology to identify the threats (possible source of damage or danger), the assets (property of the organisation or personnel categories), the risks (the likelihood of the threats actually happening to business assets), the impacts (the results of the assets and the business that occur if a threat happens), the vulnerabilities (weaknesses which a threat may be able to act upon or exploit) and the countermeasures (means capable of reducing the elements of risk) related to the operation of an information system [KPMG-93].

On the other hand, and in addition to hypotheses, there is also reality. There are actual security flaws that have been exploited in the real world. However, the data these incidents provide is not structured at all; most of the time it is not even complete and far less detailed. Metaphorically speaking, it seems that risk analysis reviews may provide a good sketch of the skeleton of a body, whereas the analysis of security flaws may provide the description of some bones of this body.

During the last decade, the increasing interest of business and government in the information systems security issues has led to - among others - the selected recordings of a large number of security incidents. Some of these inventories pertain to specific environments (e.g. computer viruses and worms, network security incidents, health information security incidents etc.). These inventories provide important data on how an intruder acts, when and how a system is breached, where an attacker aimed at, what deficiency of the information system was exploited, etc.

In order for these inventories to be effectively used by security experts or information system developers, the data they include should be first analysed. Recent reports [Lan-93] have provided some means for such analyses. According to them, a first processing should classify the security flaws, using a proper taxonomy scheme. Such a taxonomy scheme should be, for obvious reasons, as generic as possible.

Classification is usually carried out in inventories which include incidents identified and described in information systems of a similar purpose (e.g. accounting IS, health IS, banking IS, etc.). In case a risk analysis review has been also conducted in such an information system, the results of the classification could be used to support further or put in question the results of this review. It is expected that this comparison may, in principle:

- Support further a number of the risk analysis review results.

- Identify potential incompatibilities with the risk analysis review results, and perhaps lead to further investigation.

- Justify expenditures for the implementation of some very expensive countermeasures proposed by the risk analysis review [Pfl-89].

- Support the idea of changing the weights used by the risk analysis methods.

- Identify new threats with a high impact. 
In this paper, an inventory pertaining to health information systems security flaws will be processed and commented upon. This inventory has been developed by the Audit Commission for Local Authorities and the National Health Service in England and Wales, in the United Kingdom [NHS-90]. The cases included in this inventory cannot - according to its developers be considered as a representative random sample of all known flaws in this sector. However, it is considered as a useful sample due to three reasons. First, because of the extensive number of security flaws it contains (180 cases); second, because all these cases pertain to the same country (United Kingdom) and have occurred in a time span of three years (1988-90); third, because the proliferation of automated health information systems in the United Kingdom is considered to be well above average, in comparison to several other countries.

Finally, it should be noted that the cases reported in this inventory concern only to deliberate acts of fraud and abuse, and they do not include instances of accidental damage caused by fire, flood etc.

The main objective of this paper is to classify the security flaws included in this inventory according to a proper taxonomy scheme, and to draw generic conclusions, based on this classification. These conclusions might be:

- Used to focus efforts to remove security flaws and to prevent their introduction/ exploitation.

- Compared to the results of the risk analysis reviews that have been conducted in this sector, in order to strengthen the reliability of these results, or to indicate issues which require further investigation.

\section{SECURITY FLAW CLASSIFICATION}

The taxonomy scheme which will be used in this paper has been proposed in [Lan-93]. It can be used to classify a security flaw according to how, when, and where it was introduced into an automated information system.

According to [Lan-93], a given case may reveal more than one kind of security flaw. Therefore, the taxonomy does not partition the set of possible flaws. However, the same flaw may show up more than once in each of the proposed classes. Within each kind of security flaw, divisions and subdivisions are provided. Where feasible, these subdivisions are mutually exclusive and collectively exhaustive.

In the sequel, the security flaws are classified according to genesis, time of introduction, and location. The next sub-sections provide descriptions of these main flaw classifications, as well as of their divisions and subdivisions.

\subsection{Flaw classification by genesis}

2.1.1 Application of the taxonomy scheme

The first part of the proposed classification scheme is used to denote the genesis of a flaw, i.e. how a flaw was introduced into an automated information system.

In order to do so, thirteen classes are introduced. Some of these classes are grouped together to build divisions and subdivisions. In detail, the flaw classification by genesis includes the following:

1. Intentional: Includes flaws that have been intentionally introduced into a system. 
1.1 Malicious: Includes Trojan horses, trapdoors and logic/time bombs.

1.1.1 Trojan horse: A Trojan horse refers to a program that masquerades as a service, but exploits rights of a user in an unintended way.

1.1.1.1 Non-replicating: Includes Trojan horses that exploit the rights of an application user, but do not replicate themselves into another application.

1.1.1.2 Replicating: Includes Trojan horses that replicate themselves by copying their code onto other files (virus), or by creating new processes or files to contain their code (worm).

1.1.2 Trapdoor: It is a hidden piece of code that responds to a special unit, allowing its user access to resources without passing through the normal security enforcement mechanism.

1.1.3 Logic/time bomb: It is a piece of code that remains dormant in the host system until a certain "detonation" time or event occurs.

1.2 Non-malicious: Includes storage and timing covert channels, as well as other kinds of similar security flaws.

1.2.1 Covert channel: Includes paths used to transfer information in a way not intended by the system designer.

1.2.1.1 Storage: Includes covert channels that transfer information through the setting of bits by one program and through the reading of those bits, by another.

1.2.1.2 Timing: Includes covert channels that convey information by modulating some aspect of system behaviour and infer protected information.

1.2.2 Other: Includes functional requirements that are written without regard to security issues.

2. Inadvertent: Includes flaws that have been accidentally introduced into a system.

2.1 Validation error: Includes flaws that occur when a program fails to check that the parameters supplied by or returned to it conform to its assumptions about their value.

2.2 Domain error: Includes flaws that occur when the intended boundaries between protection environments have "gaps".

2.3 Serialisation/aliasing: Such a flaw permits the asynchronous behaviour of different system components to be exploited in order to cause a security violation.

2.4 Inadequate identification/authentication: Such a flaw permits a protected operation to be invoked without sufficiently checking the identity and authority of the invoking agent.

2.5 Boundary condition violation: Include flaws that reflect omission of checks, which are used to assure that constraints are not violated.

2.6 Other exploitable logic error: Includes flaws that can be invoked by users which might cause other system crashes, but that do not involve boundary conditions.

According to this classification, the results derived from the selected cases are shown in Table 1. 


\begin{tabular}{|c|c|c|c|c|c|}
\hline \multicolumn{5}{|c|}{ Flaws by genesis } & \multirow{2}{*}{$\begin{array}{c}\text { Count } \\
0\end{array}$} \\
\hline \multirow{13}{*}{ Genesis } & \multirow{7}{*}{ Intentional } & \multirow{4}{*}{ Malicious } & \multirow{2}{*}{ Trojan hor } & $\begin{array}{l}\text { Non- } \\
\text { replicating }\end{array}$ & \\
\hline & & & & Replicating & 50 \\
\hline & & & \multicolumn{2}{|c|}{ Trapdoor } & 0 \\
\hline & & & \multicolumn{2}{|c|}{ Logic/time bomb } & 3 \\
\hline & & \multirow{3}{*}{ Non-malicious } & \multirow{2}{*}{$\begin{array}{l}\text { Covert } \\
\text { channel }\end{array}$} & Storage & 0 \\
\hline & & & & Timing & 0 \\
\hline & & & \multicolumn{2}{|l|}{ Other } & 0 \\
\hline & \multirow{6}{*}{ Inadvertent } & \multicolumn{3}{|l|}{ Validation error } & 43 \\
\hline & & \multicolumn{3}{|l|}{ Domain error } & 18 \\
\hline & & \multicolumn{3}{|c|}{ Serialisation/aliasing } & 0 \\
\hline & & \multicolumn{3}{|c|}{ Inadequate identification/authentication } & 86 \\
\hline & & \multicolumn{3}{|c|}{ Boundary condition violation } & 1 \\
\hline & & \multicolumn{3}{|c|}{ Other exploitable logic error } & 1 \\
\hline \multicolumn{5}{|c|}{ Total number of flaws } & 202 \\
\hline
\end{tabular}

Table 1: Flaws by Genesis

\subsubsection{Conclusions}

The most significant conclusions that can be drawn from Table 1 are:

1. The most frequent flaw in an automated health information system is due to inadequate identification or authentication procedures. Almost 1 out of $2(42.5 \%)$ of the reported cases were due to this flaw.

2. Another frequent flaw is due to computer viruses. According to the classification, 1 out of $4(24.8 \%)$ of the reported cases were due to a computer virus or worm.

3. Validation errors cause 1 out of $5(22 \%)$ of the reported flaws.

4. Finally, domain errors caused 1 out of $9(11 \%)$ of the reported flaws. 


\subsection{Flaw classification by time of introduction}

\subsubsection{Application of the taxonomy scheme}

The second part of the proposed classification scheme is used to denote when a flaw was introduced into an automated information system, according to information system development phases.

In order to do so, five classes are introduced. Some of these classes are grouped together to build divisions and subdivisions. In detail, the flaw classification by time of introduction includes the following:

1. During development: Includes flaws introduced during the development process of the information system.

1.1 Requirement/specification phase: Includes flaws introduced during the requirements and specification phase of the information system development process.

1.2 Source code: Includes flaws that are introduced during the programming phase.

1.3 Object code: Includes flaws into the compilers or assemblers or interpreters, which have been used to create object code programs.

2. During maintenance: Includes flaws introduced during the maintenance of a system, mainly due to a programmer's failure to understand the system as a whole.

3. During operation: Includes flaws introduced during the operational use of an information system.

According to this classification, the results derived from the selected cases are shown in Table 2.

\begin{tabular}{|l|l|l|c|}
\hline \multicolumn{2}{|c|}{ Flaws by time of introduction } & $\begin{array}{c}\text { Coun } \\
t\end{array}$ \\
\hline \multirow{4}{*}{$\begin{array}{l}\text { Time } \\
\text { introduction }\end{array}$} & \multirow{3}{*}{\begin{tabular}{l} 
During development \\
\cline { 2 - 4 }
\end{tabular}} & Requirement/specification phase & 131 \\
\cline { 3 - 4 } & & Source code & 3 \\
\cline { 2 - 4 } & Object code & 0 \\
\cline { 2 - 4 } & During maintenance & $\mathbf{1 8 5}$ \\
\cline { 2 - 4 } & During operation & 48 \\
\hline Total number of flaws & & \\
\hline
\end{tabular}

Table 2: Flaws by Time of Introduction 


\subsubsection{Conclusions}

The most significant conclusions that can be drawn from Table 2 are:

1. The most frequent flaw of an automated health information system is introduced into the requirement/specification phase of the information system development process. According to the classification, 7 out of 10 flaws (70.8\%) were introduced in this phase.

2. Another frequent flaw is introduced during the operation of the information system. More than 1 out of 4 flaws (25.9\%) were introduced during this phase.

\subsection{Flaw classification by location}

2.3.1 Application of the taxonomy scheme

The last part of the proposed classification scheme is used to denote where a flaw was introduced with or found in an automated information system.

In order to do so, eleven classes are introduced. Some of these classes are grouped together to build divisions and subdivisions. In detail, the flaw classification by location includes the following:

1. Software: Includes flaws introduced into operating system, support software, or application software.

1.1 Operating system: Includes flaws that are introduced into the operating system programs.

1.1.1 System initialisation: Includes flaws introduced either because the operating system fails to establish the initial protection domains as specified, or because the system administrator has not specified a secure initial configuration for the system (L1).

1.1.2 Memory management: Includes flaws related to storage space, that permit one process to gain access to another (improperly), or to deny service to others (L2).

1.1.3 Process management/scheduling: Includes flaws related to CPU time, that permit one process to gain access to another (improperly), or to deny service to others (L3).

1.1.4 Device management: Includes flaws derived from errors in $\mathrm{V} / \mathrm{O}$ routines which fail to respect parameters provided to them, or routines which validate parameters stored in locations that can be altered (L4).

1.1.5 File management: Includes flaws derived from errors in the access controls which permit users to share and protect their files or in the management of the underlying files (L5).

1.1.6 Identification/authentication: Includes flaws derived from errors in the identification or authentication functions of the operating system (L6).

1.1.7 Other/unknown: Includes flaws in the operating system that cannot be classified into the above classes (L7).

1.2 Support software: Includes flaws introduced into compilers, editors, debuggers, subroutine or macro-libraries, data base management systems, etc.

1.2.1 Privileged utilities: Includes flaws introduced into to support programs with special privileges (L8).

1.2.2 Non-privileged utilities: Includes flaws introduced into support programs that have no special privileges (L9).

1.3 Application software: Includes flaws introduced into programs that have no special privileges, and are not widely shared as application software (L10). 
2. Hardware: Includes flaws introduced into the hardware level (including the design and implementation of processor hardware, micro programs, and supporting chips, as well as any other hardware or firmware functions used to realise the machine's instruction set architecture) (L11).

The results derived from the selected cases are shown in Table 3.

\begin{tabular}{|c|c|c|c|c|}
\hline \multicolumn{4}{|c|}{ Flaws by location } & Count \\
\hline \multirow{11}{*}{ Location } & \multirow{10}{*}{ Software } & \multirow{7}{*}{ Operating System } & System initialisation & 1 \\
\hline & & & Memory management & 0 \\
\hline & & & $\begin{array}{l}\text { Process } \\
\text { management/scheduling }\end{array}$ & 51 \\
\hline & & & Device management & 14 \\
\hline & & & File management & 37 \\
\hline & & & Identification/authentication & 34 \\
\hline & & & Other/unknown & 20 \\
\hline & & \multirow[t]{2}{*}{ Support } & Privileged utilities & 57 \\
\hline & & & Unprivileged utilities & 1 \\
\hline & & \multicolumn{2}{|l|}{ Application } & 5 \\
\hline & \multicolumn{3}{|l|}{ Hardware } & 12 \\
\hline \multicolumn{4}{|c|}{ Total number of flaws } & 232 \\
\hline
\end{tabular}

\subsubsection{Conclusions}

Table 3: Flaws by Location

The most significant conclusions that can be drawn from Table 3 are:

1. The most frequent location of a security flaw is the privileged utilities. According to this classification, 1 out of 4 flaws $(24.5 \%)$ is in privileged utilities.

2. Another frequent location of a flaw is the process management/scheduling. More than 1 out of $5(22 \%)$ of the reported flaws were located in these functions.

3. File management and identification/authentication procedures are also frequent locations of a flaw. Approximately 1 out of 7 flaws are located in these processes. 


\section{SECURITY FLAWS CROSS-TABULATIONS}

\subsection{Genesis vs location}

The most significant results that can be drawn from Table 4 are the following:

1. Computer viruses and worms are usually introduced in a non-specific location (L7) or into the file management functions (L5).

2. Identification/authentication flaws form a considerable reason causing a security breach when accessing privileged utilities (L8), or when using process management and scheduling functions (L3).

3. Validation errors are introduced into privileged utilities (L8) or into process management and scheduling functions (L3).

\begin{tabular}{|c|c|c|c|c|c|c|c|c|}
\hline Genesis Location & 1.3 & L4 & L.5 & 1.6 & L7 & L.8 & L 11 & Total \\
\hline Virus & 7 & 3 & 14 & 1 & 20 & 2 & & 47 \\
\hline Validation error & 15 & 1 & 5 & 11 & & 20 & & 52 \\
\hline Domain error & 9 & 1 & 5 & 7 & & 4 & 1 & 27 \\
\hline $\begin{array}{l}\text { Inadequate } \\
\text { identification/authentication }\end{array}$ & 31 & 12 & 18 & 26 & & 44 & 11 & 142 \\
\hline Total & 62 & 17 & 42 & 45 & 20 & 70 & 12 & 268 \\
\hline
\end{tabular}

Table 4: Security flaws - Genesis vs Location

\subsection{Genesis vs time of introduction}

The most significant results that can be drawn from Table 5 are the following:

1. Inadequate requirements/specification allows computer viruses and worms to infect a health information system.

2. Validation and domain errors occur due to inadequate specification/requirements.

3. Inadequate identification and authentication introduced into the specification/ requirement phase, and also during system operation form the main method a security flaw finds its way into an application.

\subsection{Location vs time of introduction}

The most significant results that can be drawn from Table 6 are the following:

1. Flaws found in process management and scheduling functions were introduced during the requirement/specification phase of a health information system.

2. Flaws found in privileged utilities were introduced during requirement/specification phase. 
3. Flaws found in identification and authentication procedures were introduced during the requirement/specification phase.

\begin{tabular}{|l|c|c|c|}
\hline $\begin{array}{c}\text { Time of introduction } \\
\text { Genesis }\end{array}$ & $\begin{array}{c}\text { Requircment/ } \\
\text { specification }\end{array}$ & $\begin{array}{c}\text { During } \\
\text { opcration }\end{array}$ & Total \\
\hline Virus & 26 & 22 & $\mathbf{4 8}$ \\
\hline Validation error & 37 & 3 & 40 \\
\hline $\begin{array}{l}\text { Domain error } \\
\text { Inadequate } \\
\text { identification } \\
\text { authentication }\end{array}$ & 21 & 4 & 25 \\
\hline \multicolumn{1}{|c|}{ Total } & 79 & 20 & 99 \\
\hline
\end{tabular}

Table 5: Security flaws - Genesis vs Time of Introduction

\begin{tabular}{|l|c|c|c|}
\hline $\begin{array}{c}\text { Time of introduction } \\
\text { Location }\end{array}$ & $\begin{array}{c}\text { Requirements } \\
\text { specification }\end{array}$ & $\begin{array}{c}\text { During } \\
\text { operation }\end{array}$ & Total \\
\hline $\begin{array}{l}\text { Process } \\
\text { management/scheduling }\end{array}$ & 46 & 11 & $\mathbf{5 7}$ \\
\hline Device management & 11 & 3 & $\mathbf{1 4}$ \\
\hline File management & 30 & 8 & $\mathbf{3 8}$ \\
\hline Identification/authentication & 23 & 7 & $\mathbf{3 0}$ \\
\hline $\begin{array}{l}\text { Other/unknown } \\
\text { Privileged utilities }\end{array}$ & 9 & 9 & $\mathbf{1 8}$ \\
\hline Hardware & 51 & 11 & $\mathbf{6 2}$ \\
\hline \multicolumn{1}{|c|}{ Total } & 10 & 12 & $\mathbf{2 2}$ \\
\hline
\end{tabular}

Table 6: Security flaws - Location vs Time of Introduction 


\section{CONCLUSIONS}

Security flaws in information systems usually differ, in both their severity and in their very nature, according to the purposes that these information systems are developed to serve.

The analysis and investigation of security flaws could be based either on hypothetical scenarios, or on actual cases. Obviously, actual cases provide usually more reliable means for scientific analysis.

However, in order to draw as general and precise conclusions as possible, an analyst must not be based on a limited number of cases. Although every case has - in principle - its own interest, it is the selective analysis of a large number of cases that provides meaningful results.

Therefore, large inventories of well-documented security flaws, which have been exploited in information systems of a similar purpose, can be effectively utilised by security experts, in order to draw conclusions which will guide information system developers towards developing more secure information systems.

Furthermore, the results drawn from such exercises can provide an effective means to support the reliability of a risk analysis review, which has been - or will be - conducted on an information system.

In this paper, a security flaw inventory compiled in the United Kingdom for health information systems, has been processed and analysed. First, the contents of this inventory have been classified according to a recently proposed taxonomy scheme. Second, a crosstabulation exercise has been conducted, based on this taxonomy. Finally, a number of conclusions have been arrived at.

These conclusions can be compared - to some extent - to the results of risk analysis reviews, which have been recently conducted in health information systems in the United Kingdom, as well as in some other European countries (Belgium, France, Greece, The Netherlands, Norway and Sweden), in order to support or question the reliability of these results. The results of these reviews are not yet available in a comprehensive form.

As a final conclusion, it can be said that the results provided by a risk analysis review in an information system, can be supported by the results of the analysis of a security flaw inventory which contains incidents that occurred in similar information systems, in order to identify the threats and vulnerabilities of the system under review, as well as to select and propose the countermeasures required to increase its security.

\section{Acknowledgements}

The first author of this paper wishes to express his sincere appreciation to Dr Carl Landwehr, of the Naval Research Laboratory (USA) for his idea to apply the proposed security flaw taxonomy to existing security-incident inventories.

\section{REFERENCES}

[Bad-89] Badenhorst K., Eloff J., "Framework of a methodology for the life cycle of computer security in an organisation", Computers \& Security, Vol. 8, no 6, pp. 433-442, 1989. 
[Bad-90] Badenhorst K., Eloff J., "Computer security methodology: Risk analysis and project definition", Computers \& Security, Vol. 9, no. 6, pp. 3390346, 1990.

[Elo-93] Eloff J., Labuschagne L., Badenhorst K., "A comparative framework for risk analysis methods", Computers \& Security, Vol. 12, No 8, pp. 597-603, 1993.

[Gri-94] Gritzalis D., Darzentas J., Katsikas S., "Risk Management in Health Information Systems: Trends and Perspectives", in Proc. of the 3rd Panhellenic Conference on Medical Informatics, Thessaloniki, May 1994 (invited lecture, in Greek).

[KPMG-93] Risk Analysis in EDI, Final report of CEC contracts 56193 \& 56269, KPMG Management Consulting, September 1993.

[Lan-93] Landwehr C., Bull A., McDermott J., Choi W., A Taxonomy of Computer Program Security Flaws with Examples, U.S. Naval Research Laboratory, NRL/FR/5542-93-9591, November 19, 1993.

[Lan-81] Landwehr C., "Formal Models for Computer Security", ACM Computing Surveys, Vol. 13, no. 3, pp. 247-278, September 1981.

[NHS-90] Survey of Computer Fraud \& Abuse, The Audit Commission for Local Authorities and the National Health Service in England and Wales, United Kingdom, 1991.

[Pfl-89] Pfleeger C., Security in Computing, Prentice-Hall International Editions, 1989. 